2 Sergio A Gomes ${ }^{1}$, Sebastien Behr ${ }^{2}$, Laurent S Garosi ${ }^{3}$, Ines Carrera ${ }^{4}$, Mike Targett ${ }^{5}$,

3 Mark Lowrie ${ }^{1}$

4

$5 \quad{ }^{1}$ Dovecote Veterinary Hospital, 5 Delven Lane, Castle Donington, Derby DE74 2LJ, UK

$6{ }^{2}$ Neurology/Neurosurgery Service, Willows Veterinary Centre and Referral Centre,

7 Solihull, UK ${ }^{3}$ CVS Teleneurology, 1 Owen road, Diss, IP22 4ER, UK ${ }^{4}$ Diagnostic

8 Imaging, Willows Veterinary Centre and Referral Centre, Solihull, UK ${ }^{5}$ School of

9 Veterinary Medicine and Science, University of Nottingham, Sutton Bonington,

10 Leicestershire, LE12 5RD, UK

11 Keywords: bacteria, spinal, infection, discitis

12 Name, address, and e-mail address of the corresponding author:

13 Sergio A Gomes DVM MRCVS, sergio.gomes@ dovecoteveterinaryhospital.co.uk,

14 Dovecote Veterinary Hospital, 5 Delven Lane, Castle Donington, Derby DE74 2LJ, UK

\title{
15 Abstract
}

16 Objectives This study describes the imaging features of feline discospondylitis on

17 magnetic resonance imaging (MRI), comparing them to computed tomography (CT) and

18 radiographic findings where available.

19 Methods Medical records of cats diagnosed with discospondylitis, presented to three

20 referring institutions. Magnetic resonance imaging, CT and radiographic features were

21 assessed by two of the authors independently.

22 Results Fourteen sites of discospondylitis were retrospectively identified in thirteen cats.

23 The L7-S1 intervertebral disc space (IVDS) was affected in 7/14 (50\%) of cases. 
24 Characteristic MRI features included a hyperintense nucleus pulposus signal on T2W $25(10 / 14,71 \%)$ and $\operatorname{STIR}(11 / 13,85 \%)$ with contrast-enhancement in all $(11 / 11)$, 26 involvement of adjacent vertebral endplates (11/14, 79\%), hyperintense neighbouring soft 27 tissue on T2W (11/14, 79\%) and STIR (10/13, 77\%) with contrast enhancement in all 28 (11/11) and presence of spondylosis deformans (10/14, 71\%). Other features included 29 narrowed or collapsed IVDS $(8 / 14,57 \%)$, contrast enhancement of vertebral bodies $(5 / 11$, $3046 \%$ ), epidural space involvement $(5 / 14,36 \%)$, compression of the spinal cord or nerve 31 roots $(5 / 14,36 \%)$, paraspinal abscessation $(3 / 14,21 \%)$ and meningeal signal intensity 32 abnormalities with contrast-enhancement $(5 / 6,83 \%)$. These latter findings may indicate 33 secondary focal meningitis.

34 Radiographs were available covering five sites (in 4 cats) and CT covering three sites (in 352 cats). Most common radiological features were collapse or narrowing of the affected 36 IVDS (80\%) and endplate erosion (60\%). No changes suggestive for discospondylitis 37 were identifiable on radiography or CT in two sites (1 cat) despite being identifiable on 38 MRI. Repeated radiography in one case did not reveal complete radiological resolution 39 following nine months of treatment.

40 Conclusions and relevance The results of this study indicate consistent MRI features of 41 feline discospondylitis that should be considered in the diagnosis of feline 42 discospondylitis. 
Introduction

44 Discospondylitis describes the infection of an intervertebral disc (discitis) and its adjacent cartilaginous vertebral end plates (spondylitis). ${ }^{1-5}$ This condition is well recognised and reported in dogs, with descriptions of its associated clinical signs, typical signalment and imaging characteristics. ${ }^{3,46}$ However, literature describing discospondylitis in cats is sparse with six individual case reports, and two cats being mentioned in a series of feline patients with spinal cord disease. ${ }^{7-13}$ Discospondylitis appears to be a rare condition in cats, more commonly identified in male cats mainly at the level of the lumbar spine. ${ }^{7-12}$ Prognosis appears guarded as four out of six reported cases died (1 case) or were euthanased (3 cases) following diagnosis. Reported imaging investigations included vertebral radiographs in every case with additional computed tomography (CT) or magnetic resonance imaging (MRI) in single cases. ${ }^{7,12}$

55 Discospondylitis in dogs can be challenging to diagnose as signs are variable and sometimes vague. Commonly described clinical signs include spinal hyperaesthesia, lethargy, reluctance to move, pyrexia, anorexia and weight loss. ${ }^{1,2,5}$ Neurological dysfunction can develop, usually secondary to abnormal osseous proliferation, empyema,

59 focal meningitis/myelitis, subluxation or pathologic fractures. ${ }^{4,5}$

60 Considering the variable and challenging clinical presentation, imaging is critical in 61 establishing a diagnosis of discospondylitis..$^{5}$ A diagnosis of discospondylitis relies on a combination of compatible clinical signs, exclusion of other painful and debilitating conditions, culture and sensitivity results, and cytology on any available biopsy material.

64 However, the clinical conundrum is that to attain a final diagnosis based on 65 histopathology and culture, imaging features need to be identified, in order to recognise 66 the need for further procedures. Moreover, blood or urine culture and sensitivity results have been reported to be negative in about $40-75 \%$ of cases of discospondylitis in dogs, 
68 with percutaneous disc aspiration yielding positive culture in $75 \%$ of dogs. ${ }^{2,3,14,15} \mathrm{~A}$

69 definitive diagnosis of discospondylitis in dogs, is therefore based on characteristic

70 imaging findings in conjunction with compatible clinical signs, ideally in the presence of

71 a positive culture result. ${ }^{2,5,16}$

72 MRI is considered the investigation method of choice in the diagnosis of discospondylitis

73 in both people and dogs. It is considered more sensitive and specific than other imaging

74 techniques, particularly in the early stages of the condition, being able to identify cases

75 not evident on conventional radiographs. ${ }^{4-6,17}$ There is limited literature reporting

76 diagnostic imaging findings of discospondylitis in cats, particularly with reference to

77 cross-sectional imaging.

78 The aim of this retrospective study is to describe the MRI features of discospondylitis in

79 a population of clinically affected cats. Radiography and CT features are discussed and

80 compared with MRI when available, in order to give stronger guidance for the imaging

81 diagnosis of feline discospondylitis.

82 


\section{Material and Methods}

\section{Animals}

86 Medical records of cats diagnosed with both presumptive and confirmed discospondylitis

87 at three referring institutions between February 2009 and April 2019 were reviewed.

88 Cases were included when presented with (1) clinical signs and history compatible with

89 discospondylitis, and (2) MRI features suggestive of infection in one or more 90 intervertebral discs, alone or in conjunction with its adjacent endplates. Compatible 91 clinical signs included a persistent presence of spinal hyperaesthesia in all cases, 92 lameness, abnormalities on neurological examination and pyrexia. Since no extensive 93 literature is available for MRI features of feline discospondylitis, an MRI diagnosis was 94 based on previously reported imaging characteristics of discospondylitis in a single feline 95 case report and in two case series of affected dogs.,6,12 An MRI diagnosis of 96 discospondylitis was considered when conformation or signal intensity of an 97 intervertebral disc space differed, when compared to their adjacent counterparts. The 98 finding of adjacent vertebral endplates, with an abnormal conformation or signal intensity 99 also supported the presence of a discospondylitis. All cases presenting an ongoing 100 suspected or proven neoplastic process and history of trauma were excluded.

\section{Imaging}

103 Cross-sectional imaging was performed under general anaesthesia. All cats underwent

104 CT using a multislice CT machine (Aquilion RXL; Toshiba Medical Systems 105 Corporation, Tokyo, Japan) and MRI using a low field 0.25 Tesla (T) permanent magnet 106 (Esaote VetMR Grande, Genova, Italy), a low field 0.4 T (Aperto MRI, Hitachi, Tokyo, 107 Japan), or a high field 1.5 T (Signa HDe, General Electric, London, UK). MRI studies 
108 included a minimum of T2-weighted (T2W) sagittal and transverse images in all cases, a

109 pre and post-contrast T1-weighted (T1W) and/or short tau inversion recovery (STIR)

110 dorsal, transverse or sagittal images in the remaining cases. Radiographic and CT studies

111 were retrieved and assessed when available.

\section{MRI features}

113 MRI features were assessed, with selection of these features being based on reports on

114 canine discospondylitis and a single feline report. ${ }^{4,6,12}$ The intervertebral disc space,

115 nucleus pulposus, adjacent endplates, vertebral bodies, overlying epidural space,

116 overlying meninges, paraspinal soft tissues and distal colon were all assessed. The MRI

117 features assessed are described in Table 1. The epidural space was assessed for presence

118 of suspected empyema or suspected inflammation of the epidural fat. ${ }^{4,18}$ Overlying

119 meninges were only assessed when high-field images were available, as it was considered

120 that low-field images did not offer enough resolution to perform this in detail. The

121 presence of a suspected paraspinal soft tissue abscessation was determined when a focal,

122 well-demarcated region, presenting a contrast enhancing rim-pattern with an iso-

123 hypointense center in $\mathrm{T} 2 \mathrm{~W}$ sequences was detected in direct contact with the affected

124 intervertebral disc space. ${ }^{19}$ Colonic distention was considered subjectively normal or

125 enlarged. Megacolon was considered if the ratio of maximum colonic diameter compared

126 to the length of L5 was of more than $1.48 .{ }^{20}$ When evidence of discospondylitis was found

127 on MRI then available radiographic and CT studies of the affected sites were evaluated.

\section{Radiographic features}

130 For each case, vertebral radiographs were evaluated if at least a lateral and a ventro-dorsal

131 projection were available. Assessed features included evidence of endplate erosion, 132 endplate sclerosis, vertebral body osteolysis, intervertebral disc space morphology 
133 (normal, narrowed or collapsed), osseous proliferation adjacent to the intervertebral disc

134 space, spondylosis and soft-tissue opacity alterations as well as any signs of vertebral

135 fracture, subluxation or shortening., ${ }^{4,7-10,12}$ Presence of the vacuum phenomenon was

136 evaluated and the vertebral region surveyed was noted. ${ }^{21}$

137

138 Computed tomographic features

139 Vertebral CT images were evaluated and assessed features included evidence of endplate

140 erosion, vertebral body osteolysis and its pattern (focal or multifocal punctate osteolysis),

141 intervertebral disc space morphology (normal, narrowed or collapsed), osseous

142 proliferation adjacent to the intervertebral disc space, endplate sclerosis, spondylosis,

143 soft-tissue attenuation alterations, and signs of vertebral fracture, subluxation or

144 shortening. ${ }^{7,14,22}$ Presence of the vacuum phenomena was evaluated and the vertebral 145 region surveyed was noted.

\section{Image assessment and imaging modality comparison}

148 All radiographs, CT and MRI scans were assessed by two of the authors (SG and ML)

149 independently. When an initial agreement was not attained, features were subsequently

150 revaluated and a consensus was reached.

151 Descriptive comparison of the three modalities was performed, detailing cases where

152 more than one modality was performed. In order to assess the capability of both 153 radiography and CT in detecting feline discospondylitis when compared with MRI, it was 154 considered that at least two radiological or CT features had to be identified in order for a 155 discospondylitis to be suspected based on these imaging modalities alone, e.g. a 156 narrowed/collapsed intervertebral disc space as well as eroded endplates. 
159 All follow-up repeated imaging studies in all modalities were retrieved if available and

160 described in detail. Resolution of radiological signs was considered if the lytic focus had

161 smoothed and disappeared, sclerotic margins had vanished and bridging of the affected

162 vertebrae was detected on follow-up radiographs. ${ }^{3}$

163

164 Results

165 Signalment

16613 cats were identified with a clinical diagnosis of discospondylitis. Breed distribution

167 included Domestic Short Hair (n=10), Maine Coon (2) and Siamese (1) with five females

168 and eight males with a mean age of 107.54 months (median 115, 12 - 168 months).

169

170 Fourteen foci of discospondylitis were identified in the 13 cats, with a single case 171 presenting with two affected sites. Discospondylitis was identified at L7-S1 in 7/14 172 (50\%), with T12-T13, T13-L1, L1-L2, L2-L3, L3-L4, L5-L6 and L6-L7 being

173 represented once. In the case with two affected sites these were L1-L2 and L5-L6.

174

Magnetic resonance imaging findings

176 High-field MRI was available for 6 cases and low-field MRI in the remaining 7 cases

177 encompassing 8 sites of discospondylitis. Within the fourteen imaged sites, one case had

178 no T1W sequences, in another case STIR sequences were not obtained, and in three cases

179 undergoing low-field MRI a contrast-study was not performed. The signal intensity and

180 contrast-enhancement features on MRI are detailed in Table 2. Intervertebral disc space

181 morphology was assessed as normal 6/14 (43\%), narrowed 6/14 (43\%) or collapsed 2/14

182 (14\%). There was no evidence of a concomitant disc herniation. Adjacent vertebral 
183 endplates were considered normal in 3/14 (21\%), eroded in 7/14 (50\%) and destroyed in

$1844 / 14$ (29\%). Vertebral body involvement was found in 6/14 cases and this was only found

185 to affect a maximum of a third of the vertebral body. Evidence of vertebral body shape

186 deformity was found in three cases and vertebral body subluxation was identified in one

187 case. The epidural space was considered to be involved in five sites with a suspicion of

188 either an empyema or a local inflammation of the epidural fat with a focal contrast

189 enhancement in 3/5 sites. Compression of the spinal cord was present in 5/14 sites (36\%),

190 subjectively classified as mild in 4 cases and severe in the remaining case. Concomitant

191 nerve root compression was observed in three cases. A region compatible with a

192 suspected abscess in the paraspinal tissues was found in three cases $(21 \%)$. Ventral

193 spondylosis deformans was found in 10/14 cases, and the colon was considered

194 subjectively enlarged in 10/13 cats with two presenting imaging features compatible with

195 megacolon. Examples of the MRI appearance of feline discospondylitis are depicted on

196 Figure 1.

197

198 Radiographic findings

199 Radiographs were available in four cases covering five discospondylitis sites. All

200 radiographs were performed concurrent with initial MRI studies, except in one case

201 covering two sites which was performed two weeks previous. The lumbar region was

202 included in all cases with the whole vertebral column being radiographed in one case.

203 Other cases surveyed the full thoracic spine to the tail (1), the thoracolumbar junction to

204 the tail (1) and from C3 to the tail (1). Evidence of endplate erosion alongside vertebral

205 body osteolysis was found in $3 / 5$ sites $(60 \%)$, intervertebral disc space was abnormal in

$2064 / 5$ sites being narrowed in two and collapsed in the remaining two. A single occurrence

207 was found of the following findings: endplate sclerosis, spondylosis, soft-tissue opacity, 
208 vertebral body shortening and vertebral body subluxation. No osseous proliferation

209 adjacent to the intervertebral disc space, vertebral body fractures or vacuum phenomena

210 were identified. Based on these features, clear evidence of discospondylitis was only

211 found in $3 / 5$ sites $(60 \%)$. Examples of the radiographic appearance of feline

212 discospondylitis are depicted on Figure 2.

213

214 Computed tomography findings

215 Computed tomography was performed in two cases covering three discospondylitis sites.

216 In one case the whole vertebral column was imaged whilst the other included the area of

217 interest encompassing $\mathrm{T} 7$ to the tail. Evidence of endplate erosion was present in one case

$218(33 \%)$ and intervertebral disc space morphology was considered normal in one site and

219 collapsed in the other two sites (66\%). A single occurrence was found of the following

220 findings: endplate sclerosis, spondylosis deformans and vacuum phenomena within the

221 affected intervertebral disc. No evidence of soft-tissue attenuation, osseous proliferation,

222 vertebral body osteolysis, shortening, fractures or subluxations were identified. Based on

223 these features, clear evidence of discospondylitis was only found in $1 / 3$ sites (33\%) (Table

224 3). Examples of CT appearance of feline discospondylitis are depicted on Figure 3.

225

226 Comparison of imaging modalities

227 When comparing radiographic and MRI findings in five available sites (Table 3), two

228 sites were not clearly apparent radiographically, since although one of the disc spaces was

229 collapsed, no evidence of endplate erosion or other associated features were identifiable

230 in either of them. This occurred in the case in which two discospondylitis foci were

231 identified on MRI, which was the only case where the three imaging modalities were

232 performed. Radiographs in this case were performed two weeks previous to MRI study. 
233 In these sites, the nucleus pulposus was T2W isointense, STIR hyperintense, with diffuse

234 contrast-enhancement and paraspinal tissues were involved being hyperintense on both

235 T2W and STIR sequences. Also CT, performed at the time of MRI diagnosis, did not

236 suggest discospondylitis due to the lack of endplate or vertebral body changes.

237 Radiographs provided indication of three discospondylitis sites out of five, by revealing

238 a combination, amongst other features, of signs of endplate erosion as well as narrowing

239 or collapse of the affected intervertebral disc space. None of these later cases had a CT

240 performed.

241 The second case in which a CT was performed, there was clear evidence of endplate

242 erosion, a collapsed intervertebral disc space, endplate sclerosis, evidence of subluxation

243 and ventral spondylosis deformans (Figure 3b). This was further confirmed on MRI in

244 which a T2W hyperintense nucleus pulposus with rim-contrast enhancement was

245 identified, with a third of the vertebral body affected.

\section{Follow-up}

247 Repeated imaging studies were only available for one case, in which radiography was

248 repeated 6 and 9 months following diagnosis and a treatment protocol with antibiotics

249 (Figure 4). Radiological resolution was not present: there was radiographic evidence of

250 disappearance and smoothing around a lytic focus, partial replacement by bridging of the

251 involved vertebrae however sclerotic margins were still detectable on both follow-up

252 radiographs. 


\section{Discussion}

255 This report describes the MRI features of discospondylitis in a population of cats, including its comparison with radiography and CT when available. This study revealed a series of imaging features which could aid in the detection of discospondylitis in cats.

Feline discospondylitis had been previously reported in six individual case reports and

260 two cats being described in a series of feline patients with spinal cord disease. ${ }^{7-13}$

261 Previously reported affected disc spaces in these cats were L7-S1 (3), L3-L4 (2), L4-L5

262 (2) and L2-L3 (1), with two cats presenting multiple affected discs. This study confirms

263 the suspicion that L7-S1 seems to be an intervertebral disc particularly susceptible to

264 discospondylitis in cats, making up 50\% of our reported population and making up almost

265 half of the totality of reported cases. The L7-S1 intervertebral disc space is also described as the most commonly affected site in dogs. ${ }^{2-4}$ We also report the first two instances of feline thoracic discospondylitis (T12-T13 and T13-L1).

MRI features of discospondylitis in dogs have been described previously and have been found to be generally consistent, although individual variability has been reported. ${ }^{4-6}$ In the sole feline discospondylitis report with MRI findings, the intervertebral disc was T2W hyperintense and T1W isointense, and the vertebral endplates were $\mathrm{T} 2 \mathrm{~W}$ and $\mathrm{T} 1 \mathrm{~W}$ hypointense. ${ }^{12}$ Marked contrast-enhancement of the L7 and S1 endplates and surrounding soft tissues was evident. A subjectively distended distal colon was also reported.$^{12}$

Magnetic resonance features of discospondylitis in cats appeared to be fairly consistent within the population described in this study, although individual variability was apparent. Intervertebral disc space morphology was altered in $57 \%$ of cases. Nucleus 
pulposus signal was found to be mainly hyperintense on both T2W and STIR sequences with signal void occasionally seen on T2W images. T1-weighted sequences were typically isointense, contrast uptake was noticeable in every case where this was available. Affected vertebral endplates were irregularly eroded or completely destroyed. Vertebral bodies were mostly unaffected, with the majority failing to enhance following intravenous contrast injection. The neighbouring soft tissues were often abnormal, with T2W and STIR hyperintensity and contrast enhancement present in every case where this was available. These MRI findings were mostly compatible with the MRI features described for dogs. ${ }^{4-6}$ In contrast, epidural space involvement and compression of the spinal cord or nerve roots was found in $36 \%$ of cases (5/14), which differs from dogs where both were found more commonly.,6 Overlying meningeal signal intensity

290 abnormalities were common with contrast-enhancement present in all five cases, indicating that discospondylitis in cats relates to a secondary focal meningitis. Other findings were the presence of areas compatible with paraspinal abscessation in $21 \%$ of cases, and a high prevalence of ventral spondylosis deformans (71\%).

294 Radiographic features previously described in feline patients included vertebral endplate

295 lysis and /or sclerosis, a narrowed or collapsed intervertebral disc space, spondylosis

296 deformans, irregular bone proliferation ventrally to the affected disc, an increase in 297 ventral soft-tissue opacity, and subluxation at the level of the L7-S1 joint. All of these 298 features, except for bone proliferation, were found in our population of cats. Vertebral 299 body shortening is a new feature associated with discospondylitis in our subset of patients.

300 The most common radiographic feature was collapse or narrowing of the affected 301 intervertebral disc space $(80 \%)$, with endplate erosion seen in $60 \%$ of radiographs. 302 Radiographic evidence of intervertebral disc space narrowing has been reported in cats 303 suffering from other conditions such as intervertebral disc disease and acute non- 
304 compressive nucleus pulposus extrusion. ${ }^{23-25}$ However, when evidence of intervertebral

305 disc space narrowing is identified in a cat with spinal hyperaesthesia, particularly in the 306 presence of endplate erosion, discospondylitis should be included in the list of differential

307 diagnoses. Interestingly in one of the cases previously reported, discospondylitis was 308 identified post-mortem, and had not been identified on either survey radiographs or 309 myelography. ${ }^{11}$ In our population there were two affected sites in which radiography and

310 CT failed to reveal characteristics relating to discospondylitis when changes were present 311 on MRI. In dogs, there is a reported delay in development of radiographic signs with

312 additional cross-sectional imaging often necessary to make a diagnosis. ${ }^{5}$ The presence of 313 discospondylitis with minimal or no changes on radiographs and CT, would support the 314 same assertion in feline patients. However, further cases might be required to confirm this 315 in view of the small number of cases having had all imaging modalities.

317 Computed tomography findings of discospondylitis in both cats and dogs include the 318 same features as plain radiography with the addition of being able to identify areas of 319 punctate osteolysis within the endplates with or without osteolysis of the adjacent 320 bone. $^{7,14,22}$ In one previously reported cat, contrast CT identified a rim contrast321 enhancement mass compatible with an abscess next to the affected disc. ${ }^{7}$ Computed tomography has clear advantages over plain radiography offering a more detailed 323 depiction of bone with the potential of identifying osseous lesions earlier in the course of 324 disease. ${ }^{5}$ However, in one of our cases there was a time-lapse of two weeks between 325 radiography and both CT and MRI. In this case there was no evidence of changes on 326 radiography besides a reduced intervertebral disc space. An argument could be made that 327 radiological features had not yet developed, however a CT performed at the same time as 328 MRI also failed to detect radiological features supportive of discospondylitis (Figure 3, 
$\mathrm{a}_{1}$ and $\mathrm{a}_{2}$ ). In our population of cats, CT findings were compatible with previous reports,

330 with a reduced intervertebral disc space being the most repeatable finding. Interestingly

331 the vacuum phenomenon was identified within one of the affected intervertebral discs.

332 This is a radiographic feature most commonly associated with intervertebral disc

333 extrusion. ${ }^{26}$ This is the first reported occurrence of this sign in a feline discospondylitis

334 patient, although it has previously been reported in canine discospondylitis. ${ }^{21}$ Although

335 radiography lacked sensitivity for the detection of discospondylitis, CT also failed to

336 identify discospondylitis detected on MRI in two out of the three imaged sites. Further

337 studies utilising CT in feline discospondylitis would be required to further assess its

338 potential diagnostic value.

340 The presence of infectious processes of the vertebral column in cats, such as empyema,

341 have previously been reported in cats in the absence of a concurrent discospondylitis. ${ }^{27-}$

$342{ }^{30}$ Feline discospondylitis, however, has been reported concomitantly to paravertebral

343 abscesses and meningomyelitis. ${ }^{7,9,11}$ Within our population, the subset of patients

344 presenting contrast-enhancing regions within the epidural space, meninges or paraspinal

345 soft tissues could have presented with abscessation or even meningomyelitis. When such

346 regions were identified in the epidural space, these were considered to either be a sign of

347 an empyema or inflammation of the epidural fat. The presence of these concomitant and

348 adjacent infectious loci could be explained by the close proximity of these structures

349 allowing direct spread of an infectious agent.

350

351 Imaging evidence of a subjectively enlarged colon was found in the majority of cases,

352 with megacolon found in $15 \%$. Although some faecal retention is to be expected in cases

353 presenting with spinal pain, the clinical significance of this later finding is unknown. 
354 Further clarification would require further studies describing clinical presentation and

355 treatment of feline discospondylitis and other spinal cord disorders.

356

357 Follow-up imaging was only available in one case with repeated radiographs six and nine 358 months following diagnosis. In dogs, evidence of radiological resolution of 359 discospondylitis was only achieved following treatment for a period of $53.7 \pm 45.4$

360 weeks. ${ }^{3}$ In our case there was evidence of a partial resolution of the radiological signs at 361 nine months. Further studies will be required to demonstrate if radiological resolution in

362 the feline population is similar to that reported in dogs. Follow-up cross-sectional 363 imaging, particularly MRI, may have the potential to predict clinical resolution, treatment 364 length and relapse in both feline and canine discospondylitis.

366 A number of limitations exist in the current study. Data were collected retrospectively, 367 and therefore imaging acquisition protocols and equipment were not standardised.

368 Diagnosis of discospondylitis relied on clinical features and MRI evidence of a suspected 369 infectious process affecting the intervertebral disc spaces and/or the vertebral endplates.

370 Therefore MRI was utilised as an inclusion criteria and it could therefore not be compared 371 in terms of sensitivity and specificity with the other imaging modalities. There may have 372 been cases in which MRI did not reveal any changes where a diagnosis of discospondylitis 373 could have been missed. However, imaging is critical in making a diagnosis of 374 discospondylitis and even if no abnormalities are found at an initial MRI, these should 375 develop as the condition progresses. ${ }^{5}$ A full vertebral column study was not performed in 376 most cases, leaving the potential for other affected intervertebral discs being overlooked.

377 This can be explained by costs associated with advanced imaging and investigation based 378 on an area of interest identifiable either through a clear neurolocalisation or an indication 
of a neurolocalisation based on spinal hyperaesthesia. We would recommend that when

380 a focus of discospondylitis is detected, imaging of the entire vertebral column is

381 performed in search of other possible foci of infection. Only a small number of

382 radiographs and CT studies were available in relation with MRI studies, which limited

383 the comparison within modalities. Follow-up study was only available in one case and

384 further information could have been gathered with an increased number of cases.

385

386 Conclusions

387 This is the largest reported population of cats diagnosed with discospondylitis. A set of 388 MRI features are described, indicating a series of consistent findings that might be helpful 389 in the diagnosis of discospondylitis in cats. Although only a few cases had all imaging 390 modalities performed, the findings in this study support the notion that MRI should be 391 considered the investigation method of choice in the diagnosis of discospondylitis in 392 feline patients, as is presently considered in both dogs and humans. Where only 393 radiography is available, evidence of intervertebral disc space narrowing in conjunction

394 with adjacent endplate irregularities should be considered a strong indication for the 395 presence of discospondylitis, and further advanced imaging should be performed.

397 Conflict of interest

398 The authors declared no potential conflicts of interest with respect to the research, 399 authorship, and/ or publication of this article.

400

\section{$401 \quad$ Funding}

402 The authors received no financial support for the research, authorship, and/or publication 403 of this article. 
$405 \quad$ Ethical approval

406 This work involved the use of client-owned animals only, and followed established

407 internationally recognised high standards ('best practice') of individual veterinary clinical 408 patient care. Ethical Approval from a committee was not therefore needed.

409

410 Informed consent

411 Written informed consent was obtained from the owner or legal guardian of all animals 412 described in this work for the procedures undertaken.

413 
4151 Moore MP. Discospondylitis. Vet Clin North Am Small Anim Pract 1992; 22(4):

$416 \quad 1027-1034$.

4172 Thomas WB. Diskospondylitis and other vertebral infections. Vet Clin North Am $418 \quad$ Small Anim Pract 2000; 30(1): 169-82.

4193 Burkert AB, Kerwin SC, Hosgood GL, et al. Signalment and clinical features of 420 diskospondylitis in dogs: 513 cases (1980-2001). J Am Vet Med Assoc 2005; 227: $421 \quad 268-275$.

4 Carrera I, Sullivan M, McConnell F, et al. Magnetic resonance imaging features of 423 discospondylitis in dogs. Vet Radiol Ultrasound 2011; 52: 125-131.

4245 Ruoff CM, Kerwin SC and Taylor AR. Diagnostic imaging of discospondylitis. Vet 425 Clin North Am Small Anim Pract 2018; 48(1): 85-94.

4266 Harris JM, Chen AV, Tucker RL, et al. Clinical features and magnetic resonance 427 imaging characteristics of diskospondylitis in dogs: 23 cases (1997-2010). ). J Am $428 \quad$ Vet Med Assoc 2013; 242(3): 359-365.

4297 Packer RA, Coates JR, Cook CR, et al. Sublumbar abscess and diskospondylitis in a $430 \quad$ cat. Vet Radiol Ultrasound 2005; 46: 396-399.

4318 Norsworthy GD. Discospondylitis as a cause of posterior paresis. Feline Pract 9:3943240,1979

4339 Malik R, Latter M and Love DN. Bacterial discospondylitis in a cat. J Small Anim $434 \quad$ Pract 1990; 31: 404-406.

43510 Watson E, Roberts RE. Discospondylitis in a cat. Vet Radiol Ultrasound 1993; 34: $436 \quad 397-398$.

43711 Aroch I, Shamir M and Harmelin A. Lumbar diskospondylitis and meningomyelitis 438 caused by Escherichia coli in a cat. Feline Pract 1999; 27: 20-22. 
12 Hill MF, Warren-Smith C and Granger N. What is your diagnosis?

440 Diskospondylitis. J Am Vet Med Assoc 2015; 247: 743-745. [isp]

13 Gonçalves R, Platt SR, Llabrés-Díaz FJ, et al. Clinical and magnetic resonance imaging findings in 92 cats with clinical signs of spinal cord disease. J Feline Med Surg 2009; 11: 53-59.

14 Gonzalo-Orden JM, Altonaga JR, Asuncion Orden M et al. Magnetic resonance, computed tomographic and radiologic findings in a dog with discospondylitis. Vet Radiol Ultrasound 2000; 41(2), 142-144.

15 Fischer A, Mahaffey MB and Oliver JE. Fluoroscopically Guided Percutaneous Disk Aspiration in 10 Dogs With Diskospondylitis. J Vet Intern Med 1997; 11(5): 284-287.

16 Tipold A, Stein VM. Inflammatory Diseases of the Spine in Small Animals. Vet Clin North Am Small Anim Pract 2010; 40(5): 871-879.

17 Maiuri F, Gallicchio B, Manto A, et al. Spondylodiscitis: clinical and magnetic resonance diagnosis. Spine 1997; 22(15): 1741-1746.

18 Cornelis I, De Decker S, Gielen I, et al. Idiopathic sterile inflammation of the epidural fat and epaxial muscles causing paraplegia in a mixed-breed dog. J Am Vet Med Assoc 2013; 242(10): 1405-1409.

19 Naughton JF, Tucker RL and Bagley RS. Radiographic diagnosis - paraspinal abscess in a dog. Vet Radiol Ultrasound 2005; 46(1): 23-26.

20 Trevail T, Gunn-Moore DA, Carrera I, et al. Radiographic diameter of the colon in 460 normal and constipated cats and in cats with megacolon. Vet Radiol Ultrasound

46221 Weber WJ, Berry CR and Kramer RW. Vacuum phenomenon in twelve dogs. Vet Radiol Ultrasound 1995, 36(6), 493-498. 
22 De Risio L, Gnudi G and Bertoni G. What is your diagnosis? Sclerosis of the caudal 465 vertebral body end plate of L7 and the cranial end plate of S1 and narrowing of the 466 L7-S1 intervertebral disk space. J Am Vet Med Assoc 2003; 222: 1359-60.

46723 Kathmann I, Cizinauskas S, Rytz U, et al. Spontaneous Lumbar Intervertebral Disc 468 Protrusion in Cats: Literature Review and Case Presentations. J Feline Med Surg $469 \quad 2000 ; 2(4): 207-212$.

47024 Knipe M, Vernau K, Hornof W, et al. Intervertebral Disc Extrusion in Six Cats. $J$ Feline Med Surg 2001; 3(3), 161-168.

25 Chow K, Beatty JA, Voss K, et al. Probable lumbar acute non-compressive nucleus pulposus extrusion in a cat with acute onset paraparesis. J Feline Med Surg 2012; 14(10): 764-767.

26 Müller MK, Ludewig E, Oechtering G, et al. The vacuum phenomenon in intervertebral disc disease of dogs based on computed tomography images. J Small Anim Pract 2013; 54(5): 253-257.

27 Granger N, Hidalgo A, Leperlier D, et al. Successful treatment of cervical spinal epidural empyema secondary to grass awn migration in a cat. J Feline Med Surg

28 Maeta N, Kanda T, Sasaki T, et al. Spinal epidural empyema in a cat. J Feline Med 482 Surg 2010; 12(6): 494-497.

29 Rapoport K, Shamir MH, Bibring U, et al. Epidural Spinal Empyema and Vertebral Osteomyelitis in a Cat. ISR J VET MED 2016; 71(4): 41-44.

30 Guo S, Lu D. Clinical presentation, diagnosis, treatment and outcome of spinal epidural empyema in four cats (2010 to 2016). J Small Anim Pract. Epub ahead of print 02 November 2018. 
Table 1. MRI features assessed

492

\begin{tabular}{|c|c|}
\hline Region of interest & MRI features based on Carrera et al. 2010 and Harris et al. 2013 \\
\hline \multirow[t]{4}{*}{ Intervertebral disc space (IVDS) } & Number and location of affected intervertebral discs. \\
\hline & Morphology (normal, narrowed or collapsed in comparison with contiguous IVDS). \\
\hline & Presence of intervertebral disc herniation. \\
\hline & Presence of ventral spondylosis deformans. \\
\hline \multirow[t]{2}{*}{ Intervertebral disc nucleus pulposus } & Intensity on $\mathrm{T} 2 \mathrm{~W}, \mathrm{~T} 1 \mathrm{~W}$ and STIR compared to adjacent discs. \\
\hline & Contrast-enhancement pattern (focal, diffuse, rim-enhancement or absent) \\
\hline Adjacent endplates & $\begin{array}{l}\text { Intact / eroded (hypointense signal alongside normal signal intensity of the adjacent } \\
\text { marrow) / destroyed (both cortical and adjacent marrow signal disruption) }\end{array}$ \\
\hline \multirow[t]{4}{*}{ Vertebral body } & Intensity on $\mathrm{T} 2 \mathrm{~W}, \mathrm{~T} 1 \mathrm{~W}$ and STIR compared with normal vertebral bone marrow. \\
\hline & Extent of abnormalities (one-third, two-thirds, complete). \\
\hline & Contrast-enhancement pattern (as described above). \\
\hline & Morphology (presence of deformity or subluxation). \\
\hline \multirow[t]{4}{*}{ Epidural space } & Presence of suspected empyema / epidural fat inflammation. \\
\hline & Contrast-enhancement pattern (as described above). \\
\hline & Spinal cord compression (mild, moderate, severe). \\
\hline & Nerve root compression. \\
\hline \multirow[t]{2}{*}{ Meninges } & Intensity on $\mathrm{T} 2 \mathrm{~W}, \mathrm{~T} 1 \mathrm{~W}$ and STIR. \\
\hline & Contrast-enhancement pattern (as described above). \\
\hline \multirow[t]{3}{*}{ Paraspinal tissues } & Intensity on $\mathrm{T} 2 \mathrm{~W}, \mathrm{~T} 1 \mathrm{~W}$ and STIR. \\
\hline & Contrast-enhancement pattern (as described above). \\
\hline & Suspected abscess presence. \\
\hline Colonic distention & Normal, enlarged or megacolon. \\
\hline
\end{tabular}




\section{7 found in this study. The most frequent finding in each category is highlighted}

\begin{tabular}{|c|c|c|c|c|}
\hline & T2-weighted & T1-weighted & STIR & $\begin{array}{l}\text { T1-weighted post- } \\
\text { contrast pattern or } \\
\text { presence }\end{array}$ \\
\hline $\begin{array}{l}\text { Intervertebral } \\
\text { disc nucleus } \\
\text { pulposus }\end{array}$ & $\begin{array}{c}\text { Hyperintense 10/14 } \\
(71 \%) \\
\text { Isointense 1/14 (7\%) } \\
\text { Hypointense 2/14 (14\%) } \\
\text { Not identifiable 1/14 } \\
(7 \%)\end{array}$ & $\begin{array}{c}\text { Hyperintense } 0 / 13 \\
(0 \%) \\
\text { Isointense } 9 / 13(69 \%) \\
\text { Hypointense } 3 / 13 \\
(23 \%) \\
\text { Not identifiable } 1 / 13 \\
(8 \%) \\
\end{array}$ & $\begin{array}{c}\text { Hyperintense 11/13 } \\
(85 \%) \\
\text { Isointense } 1 / 13(8 \%) \\
\text { Hypointense } 0 / 13(8 \%) \\
\text { Not identifiable } 1 / 13 \\
(8 \%)\end{array}$ & $\begin{array}{c}\text { Absent 0/11 }(0 \%) \\
\text { Focal 2/11 (18\%) } \\
\text { Diffuse 6/11 (55\%) } \\
\text { Rim-like 3/11 (27\%) }\end{array}$ \\
\hline Vertebral body & $\begin{array}{l}\text { Hyperintense 0/14 }(0 \%) \\
\text { Isointense } 11 / 14(79 \%) \\
\text { Hypointense } 3 / 14(21 \%)\end{array}$ & $\begin{array}{c}\text { Hyperintense } 0 / 13 \\
(0 \%) \\
\text { Isointense } 9 / 13(69 \%) \\
\text { Hypointense } 4 / 13 \\
(31 \%) \\
\end{array}$ & $\begin{array}{c}\text { Hyperintense } 3 / 13 \\
(23 \%) \\
\text { Isointense } 10 / 13 \\
(77 \%) \\
\text { Hypointense } 0 / 13(0 \%) \\
\end{array}$ & $\begin{array}{c}\text { Absent 6/11 (55\%) } \\
\text { Focal 5/11 (46\%) } \\
\text { Diffuse 0/11 (0\%) } \\
\text { Rim-like 0/11 (0\%) }\end{array}$ \\
\hline $\begin{array}{l}\text { Paraspinal } \\
\text { tissues }\end{array}$ & $\begin{array}{l}\text { Hyperintense } 11 / 14 \\
\quad(79 \%) \\
\text { Isointense } 3 / 14(21 \%) \\
\text { Hypointense } 0 / 14(0 \%)\end{array}$ & $\begin{array}{c}\text { Hyperintense } 0 / 13 \\
(0 \%) \\
\text { Isointense } 13 / 13 \\
(100 \%) \\
\text { Hypointense } 0 / 13(0 \%)\end{array}$ & $\begin{array}{c}\text { Hyperintense 10/13 } \\
\quad(77 \%) \\
\text { Isointense 3/13 (23\%) } \\
\text { Hypointense 0/13 (0\%) }\end{array}$ & $\begin{array}{c}\text { Absent 1/11 }(9 \%) \\
\text { Focal 3/11 }(27 \%) \\
\text { Diffuse 7/11 (64\%) } \\
\text { Rim-like 0/11 (0\%) }\end{array}$ \\
\hline $\begin{array}{l}\text { Meninges (only } \\
\text { evaluated in } \\
\text { high-field } \\
\text { imaging) }\end{array}$ & $\begin{array}{c}\text { Hyperintense } 5 / 6(83 \%) \\
\text { Isointense } 1 / 6(17 \%) \\
\text { Hypointense 0/6 (0\%) }\end{array}$ & $\begin{array}{c}\text { Hyperintense } 0 / 6(0 \%) \\
\text { Isointense } 5 / 6(83 \%) \\
\text { Hypointense } 1 / 6(17 \%)\end{array}$ & $\begin{array}{c}\text { Hyperintense } 4 / 5 \\
(80 \%) \\
\text { Isointense } 1 / 5(20 \%) \\
\text { Hypointense } 0 / 5(0 \%) \\
\end{array}$ & Present 5/6 (83\%) \\
\hline Epidural space & \multicolumn{3}{|c|}{ Found involved in 5/14 (36\%) cases } & $\begin{array}{c}\text { Absent 0/5 }(0 \%) \\
\text { Focal 3/5 }(60 \%) \\
\text { Diffuse 2/5 }(40 \%) \\
\text { Rim-like } 0 / 5(0 \%)\end{array}$ \\
\hline
\end{tabular}

498

499 Table 3. Comparison of different imaging modalities in the available cases

500

\begin{tabular}{|l|l|l|l|l|l|}
\hline & $\begin{array}{l}\text { Lesion } \\
\text { location }\end{array}$ & $\begin{array}{l}\text { MRI } \\
\text { demonstrable }\end{array}$ & $\begin{array}{l}\text { CT } \\
\text { demonstrable }\end{array}$ & $\begin{array}{l}\text { Radiographically } \\
\text { demonstrable }\end{array}$ & $\begin{array}{l}\text { Repeat } \\
\text { radiography }\end{array}$ \\
\hline Cat 1 & L7-S1 & $\sqrt{ }$ & & $\sqrt{ }$ & \\
\hline Cat 2 & L1-L2 & $\sqrt{ }$ & $\mathrm{x}$ & $\mathrm{x}$ & \\
\hline Cat 2 & L5-L6 & $\sqrt{ }$ & $\mathrm{x}$ & $\mathrm{x}$ & \\
\hline Cat 3 & L2-L3 & $\sqrt{ }$ & & $\sqrt{ }$ & \\
\hline Cat 4 & L7-S1 & $\sqrt{ }$ & & $\sqrt{ }$ & \\
\hline Cat 5 & L7-S1 & $\sqrt{ }$ & $\sqrt{ }$ & & \\
\hline
\end{tabular}

501

502 Figures

503

504 Figure 1. Three examples of feline discospondylitis on sagittal plane MRI: (a)

505 discospondylitis present at L3-L4 (arrow) acquired on high-field MRI: A 1 T2-weighted,

$506 \quad \mathrm{~A}_{2}$ T1-weighted pre-contrast, $\mathrm{A}_{3}$ T1-weighted post-contrast; (b) discospondylitis present 
at T12-T13 (arrow) acquired on low-field MRI: $\mathrm{B}_{1}$ T2-weighted, $\mathrm{B}_{2}$ T1-weighted pre-

508 contrast, $\mathrm{B}_{3}$ T1-weighted post-contrast; (c) discospondylitis present at L2-L3 (arrow)

509 acquired on high-field MRI: $\mathrm{C}_{1}$ T2-weighted, $\mathrm{C}_{2}$ T1-weighted pre-contrast, $\mathrm{C}_{3}$ T1-

510 weighted post-contrast

511

512 Figure 2. Two examples of feline discospondylitis identifiable on radiography. (a) L2-

513 L3 discospondylitis (arrow): $\mathrm{A}_{1}$ lateral projection, $\mathrm{A}_{2}$ ventro-dorsal projection. There is 514 loss of normal endplate morphology, left lateral bone proliferation (arrow) and

515 intervertebral disc space narrowing -endplate erosion with evidence of a reduced foramen

516 at this level confirms a narrower space in comparison with adjacent spaces. (b) L7-S1

517 discospondylitis (arrow): $\mathrm{B}_{1}$ lateral projection, $\mathrm{B}_{2}$ ventro-dorsal projection. There is

518 endplate destruction and sclerosis, evidence of subluxation, osteolytic lesion at the S1

519 vertebral body and collapse of the intervertebral disc space at this level. A subjectively

520 enlarged distal colon is also identifiable $(*)$

521

522 Figure 3. Two examples of feline discospondylitis identifiable on computed tomography.

523 (a) L5-L6 discospondylitis (arrow): $\mathrm{A}_{1}$ sagittal plane, $\mathrm{A}_{2}$ dorsal plane. Narrowing of the 524 intervertebral disc space is identifiable without endplate erosion. (b) L7-S1 525 discospondylitis (arrow): $\mathrm{B}_{1}$ sagittal plane, $\mathrm{B}_{2}$ dorsal plane. There is endplate sclerosis, 526 collapse of the intervertebral disc space and evidence of spondylosis deformans ventral 527 to the affected disc. A subjectively enlarged distal colon is also identifiable (*)

529 Figure 4. L7-S1 feline discospondylitis identifiable on repeated radiography following 530 treatment with antibiotics: a1 (initial), a2 (6 months later), a3 (9 months later). Full 531 radiological resolution was not present despite clinical resolution 


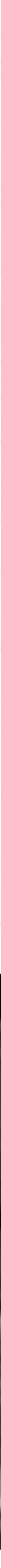


R

$\uparrow$

$A_{1}$

$A_{2}$
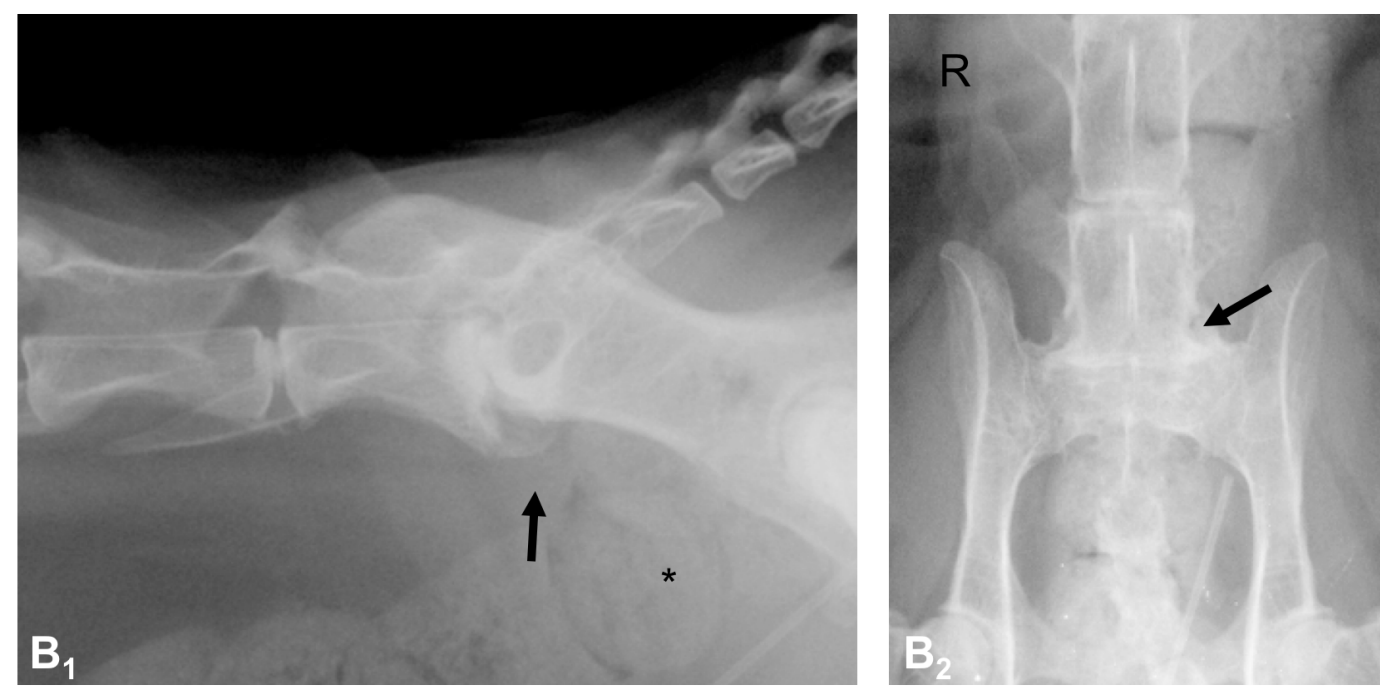


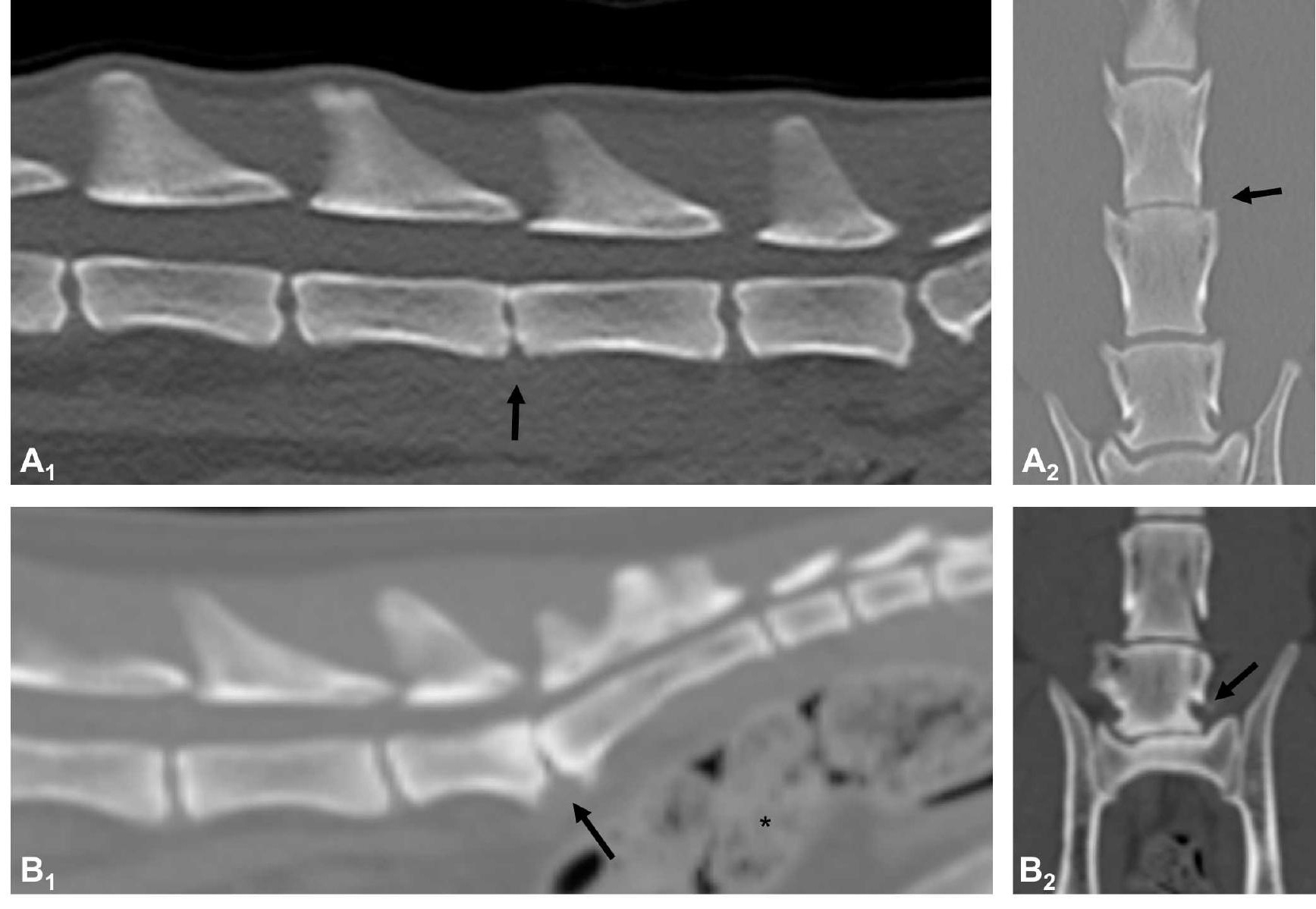


